EPJ Web of Conferences 61, 04009 (2013)

DOI: 10.1051/epjconf/ 20136104009

(C) Owned by the authors, published by EDP Sciences, 2013

\title{
On the connection between radio and gamma rays
}

\section{Variability and polarization properties in relativistic jets}

\author{
M. Orienti ${ }^{1}$, a, F. D’Ammando ${ }^{1}$, M. Giroletti ${ }^{1}$, D. Dallacasa ${ }^{2,1}$, T. Venturi ${ }^{1}$, and G. Giovannini ${ }^{2,1}$ \\ ${ }^{1}$ INAF-IRA, via Gobetti 101, 40129 Bologna, Italy \\ ${ }^{2}$ DIFA, University of Bologna, via Ranzani 1, 40127 Bologna, Italy
}

\begin{abstract}
Relativistic jets are one of the most powerful manifestations of the release of energy produced around supermassive black holes at the centre of active galactic nuclei (AGN). Their emission is observed across the entire electromagnetic spectrum, from the radio band to gamma rays. Despite decades of efforts, many aspects of the physics of relativistic jets remain elusive. In particular, the location and the mechanisms responsible for the high-energy emission and the connection of the variability at different wavelengths are among the greatest challenges in the study of AGN.

From the comparison of the radio and gamma-ray light curves of gamma-ray flaring objects, there is evidence that some flares, either in radio or in gamma rays, have not an obvious connection at the other extreme of the electromagnetic spectrum, like in the case of the Narrow-Line Seyfert 1 SBS 0846+513. An intriguing aspect pointed out by high resolution radio observations is the change of the polarization properties close in time with some high energy flares. In particular, in PKS 1510-089 and 3C 454.3 a rotation of almost 90 degrees has been observed after strong gamma-ray flares. The swing of the polarization angle may be related either to the propagation of a shock along the jet that orders the magnetic field, or a change of the opacity regime.
\end{abstract}

\section{Introduction}

The extragalactic gamma-ray sky is largely dominated by radio-loud active galactic nuclei (AGN). In particular, the population of blazars represents almost $97 \%$ of the gamma-ray emitting AGN from the 2LAC catalogue [4]. The high energy emission is likely due to inverse Compton scattering off the low energy photons by the relativistic electrons which are also responsible for the synchrotron emission observed in the radio band. Although in blazars all the ingredients needed for this scenario are present, a clear connection between the emission at the edges of the multiwavelength spectrum has not been unambiguously established yet. In particular, the trigger of the high-energy flares typically observed in blazars, and the location of the gamma-ray emitting region are still uncertain.

Thanks to their luminosity variability observed throughout the electromagnetic spectrum, we have a chance to shed a light on this issue. The variability behaviour shown in the various energy bands (i.e. timedelay, duration, intensity) provides us tight constraints on the location and size of the gamma-ray emitting region. For example, intra-day variability is an indication of a very compact region. Moreover, a time delay in the emergence of the flare at progressively longer wavelengths may locate the high-energy emitting region in the innermost part of the AGN where severe opacity effects play a role [10]. On the other hand, the detection of gamma-

\footnotetext{
ae-mail: orienti@ira.inaf.it
}

ray and millimeter-wavelength flares occurring almost simultaneously may indicate that the high-energy photons are produced much further out, downstream along the jet $[13,16]$. High-resolution observations performed with the Very Long Baseline Array (VLBA) found that superluminal jet components are ejected close in time with strong gamma-ray flares [11].

With the aim of understanding the origin of gammaray emission, multiwavelength monitoring campaigns triggered by strong high-energy flares are required. Thanks to the high sensitivity and the gamma-ray all-sky monitoring, the Large Area Telescope (LAT) on board Fermi has proved to be a superb hunter of gamma-ray flares. However, despite the efforts a clear picture is far from being drawn. From the detailed study of the most variable sources it seems that not all the flares have the same characteristics, even if produced within the same source.

In this contribution we present results on the multiwavelength variability study for PKS 1510-089, 3C 454.3, and SBS $0846+513$. These objects underwent several gamma-ray flaring episodes detected by LAT and were subject of dedicated multiwavelength campaigns. In particular these sources are part of the Monitoring Of Jets in Active galactic nuclei with the VLBA Experiment (VLBA) programme ${ }^{1}$, which provides multi-epoch polarimetric observations at $15 \mathrm{GHz}$ with a sub-milliarcsecond

\footnotetext{
${ }^{1}$ The MOJAVE data archive is maintained at http://www.physics.purdue.edu/MOJAVE.
} 
Table 1. Kinematic properties of the superluminal jet components in PKS 1510-089. Column 1: component; Col. 2: number of observing epochs considered for the linear regression fit; Cols. 3, 4: apparent angular velocity (mas/yr) and linear velocity, respectively; Col. 5: ejection time estimated from the regression fit.

\begin{tabular}{ccccc}
\hline Comp. & Ep. & $\mu$ & $\beta$ & $\mathrm{T}_{0}$ \\
\hline $\mathrm{A}$ & 10 & $1.06 \pm 0.06$ & $23.6 \pm 1.3$ & $13-09-2008$ \\
$\mathrm{~B}$ & 8 & $1.10 \pm 0.11$ & $24.5 \pm 2.4$ & $20-05-2009$ \\
$\mathrm{C}$ & 26 & $1.56 \pm 0.03$ & $34.7 \pm 0.7$ & $14-03-2010$ \\
$\mathrm{D}$ & 11 & $1.23 \pm 0.11$ & $27.4 \pm 2.4$ & $20-11-2011$ \\
$\mathrm{E}$ & 5 & $1.28 \pm 0.40$ & $28.5 \pm 8.9$ & $24-03-2012$ \\
\hline
\end{tabular}

resolution [14].

Throughout this contribution, we assume $H_{0}=71 \mathrm{~km}$ $\mathrm{s}^{-1} \mathrm{Mpc}^{-1}, \Omega_{\mathrm{M}}=0.27, \Omega_{\Lambda}=0.73$, in a flat Universe.



Figure 1. VLBA image at $15 \mathrm{GHz}$ of the parsec-scale structure of PKS 1510-089.

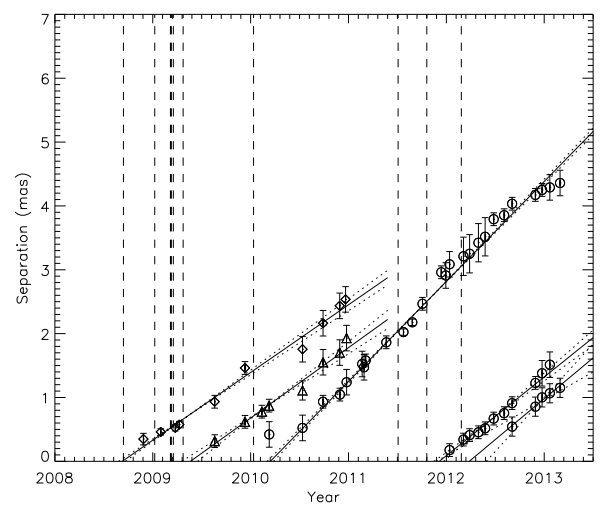

Figure 2. Changes in separation with time between the core component, considered stationary, and the new knots ejected since 2008 for PKS 1510-089. The solid line represents the regression fit to the $15-\mathrm{GHz}$ VLBA data, while the dashed lines represent the uncertainties from the fit parameters. Dashed vertical lines indicate the time of the gamma-ray flares.

\subsection{Flux density variability}

The high angular resolution of MOJAVE observations allows us to separate the emission of the core region from the contribution of the jet. Disentangling these components is crucial for locating the region where the high energy emission originates. In Fig. 3 we show the $15-\mathrm{GHz}$ light curves for the core component and the jet knots, together with their fractional polarization and the electric vector position angle (EVPA).

If we consider the properties of the radio core behaviour, no trivial correlation between radio and gammaray flares is unambiguously determined for all the episodes (three upper panels in Fig. 3). Some high-energy flares seem to precede the increase of the radio flux density, like 
those observed in April 2009 and February 2012, as a consequence of opacity effects. Other flares, like in July 2011, and those observed at the beginning of 2008, do not have any obvious relation with changes in the radio emission, suggesting that the production of high energy photons occurs in the innermost part of the AGN, which is totally opaque to the radio wavelengths.

In the case of the October 2011 flare, the high energy outburst seems to take place almost simultaneously to the millimeter flare. Such simultaneity may be an indication that high-energy photons are produced in a region which is already transparent to the radiation at millimeter wavelength, implying a location that is far away from the Broad Line Region (BLR), likely parsec scale downstream along the jet [19]. At such distances the high energy emission would be produced by inverse Compton scattering off the infrared photons of the dusty torus [20], or by synchrotron-self Compton [e.g. 16].

\subsection{Polarization properties}

\subsubsection{The unresolved core component}

No clear trend is found between the gamma-ray flares and the polarization properties. The polarization percentage in the core never exceeds $5 \%$, while in the jet knots may reach $10 \%$. An interesting aspect is shown by the electric vector position angle (EVPA), both in optical and radio band (Table 2). It has been noted that before some gammaray flares, the optical EVPA is subjected to a huge rotation (from $330^{\circ}$ to $720^{\circ}$, tracked by daily measurements) which lasts a few days and culminates when the gamma-ray flare takes place. On the other hand, the radio EVPA behaves differently during different flares. For example, although the optical EVPA rotates of about $380^{\circ}$ in the July 2011 flaring episode, no significant change in the radio EVPA has been observed. A similar behaviour was found in BL Lacertae and it was interpreted in terms of a shock, produced in the very initial part of the jet totally opaque to the radio wavelengths, which follows a spiral path as it moves through a toroidal magnetic field [15].

Interestingly, after the February 2012 flare, which was detected up to very-high-energy (VHE) by MAGIC [5] the radio EVPA showed a smooth rotation of about $80^{\circ}$ in 2 months, rather than an abrupt flip, as the one detected after the other VHE flare that occurred in March and April 2009 and detected by HESS [2], when the radio EVPA rotated of about $70^{\circ}[18]$.

\subsubsection{The jet knots}

The high spatial resolution allows us to follow and study the properties of the various ejected knots as they move along the jet. No correlation is found between the jet properties and the gamma-ray flares, indicating that the highenergy emission does not arise from the knot itself, but it is likely located in the unresolved core component.

From Fig. 3 it is possible to identify three knots and follow how their emission evolves as they propagate

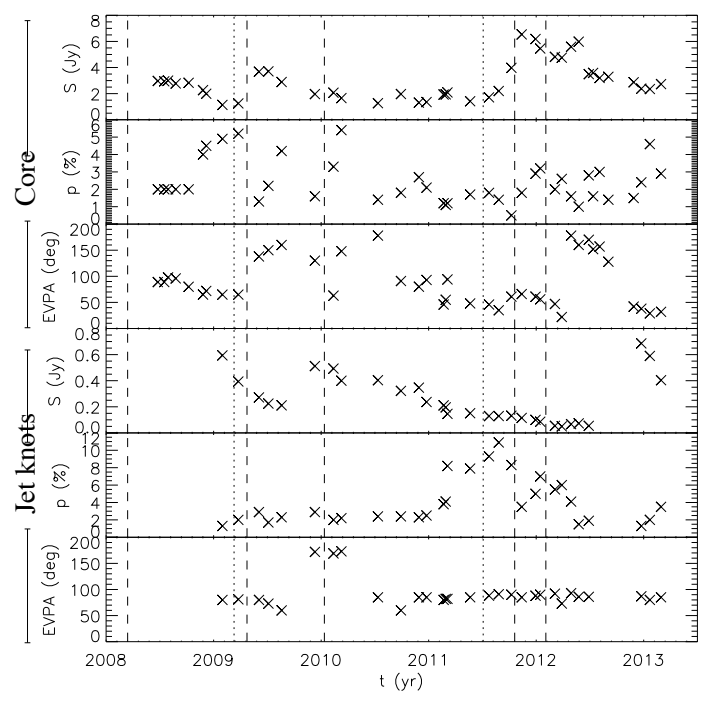

Figure 3. Total intensity flux density, fractional polarization, and polarization angle for the core component (upper panels) and the ejected knots (lower panels) in PKS 1510-089. Vertical dashed lines and dotted lines represent gamma-ray flares with or without the emergence of a new jet component, respectively.

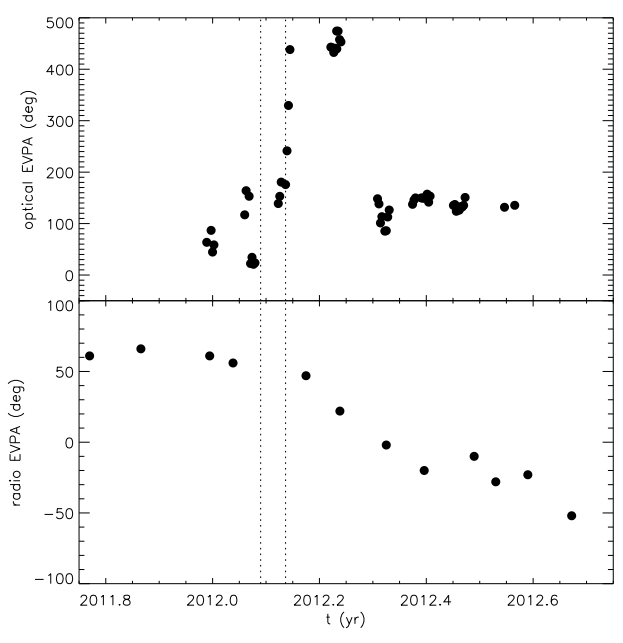

Figure 4. Optical EVPA (top) and radio EVPA (bottom) in PKS $1510-089$. Multiples of $180^{\circ}$ are added to EVPA as needed to minimize the jumps in consecutive values. The area between the vertical lines represents the period of the VHE detection by MAGIC [5]. Optical data are from the Steward Observatory blazar monitoring programme of the University of Arizona. A description of this monitoring project, the calibration and the data products can be found in [21].

along jet. The flux density decreases as expected for adiabatic losses, and the polarization percentage increases reaching values up to $10 \%$, much larger than what is found in the core component. Remarkably, all the knots are characterized by the same EVPA $\sim 80^{\circ}$, with some exceptions likely due the contamination from another blended component (Orienti et al., in preparation). 
Table 2. Summary of the gamma-ray flares observed in PKS 1510-089. Column 1: flare number; Cols. 2 and 3: date of the gamma-ray flare and the Astronomer's Telegram reporting the detection; Col. 4: change in the radio EVPA, from MOJAVE data; Col. 5: change in the optical EVPA with the reference: $a=[16] ; b=[19] ; c=$ this contribution (Orienti et al., in prep.); Col. 6: ejection of a superluminal knot close in time; Col. 7: VHE detection and reference: $d=[2] ; e=[5]$.

\begin{tabular}{ccccccc}
\hline Flare & Date & $\begin{array}{c}\text { ATel } \\
\text { N. }\end{array}$ & $\begin{array}{c}\Delta \text { EVPA } \\
\text { radio }\end{array}$ & $\begin{array}{c}\Delta \text { EVPA } \\
\text { optical }\end{array}$ & knot & VHE \\
\hline 1 & $13 / 09 / 2008$ & $\# 1743$ & $<15^{\circ}$ & - & Yes & - \\
2 & $09 / 03 / 2009$ & $\# 1957$ & $<10^{\circ}$ & - & No & Yes, d \\
3 & $25 / 04 / 2009$ & $\# 2033$ & $\sim 70^{\circ}$ & $720^{\circ}$, a & Yes & Yes, d \\
4 & $11 / 01 / 2010$ & $\# 2385$ & $<10^{\circ}$ & - & Yes & - \\
5 & $04 / 07 / 2011$ & $\# 3473$ & $<10^{\circ}$ & $380^{\circ}, \mathrm{b}$ & No & - \\
6 & $19 / 10 / 2011$ & $\# 3694$ & $<15^{\circ}$ & - & Yes & - \\
7 & $28 / 01 / 2012$ & $\# 3907$ & $\sim 80^{\circ}$ & $330^{\circ}, \mathrm{c}$ & Yes & Yes, e \\
\hline
\end{tabular}

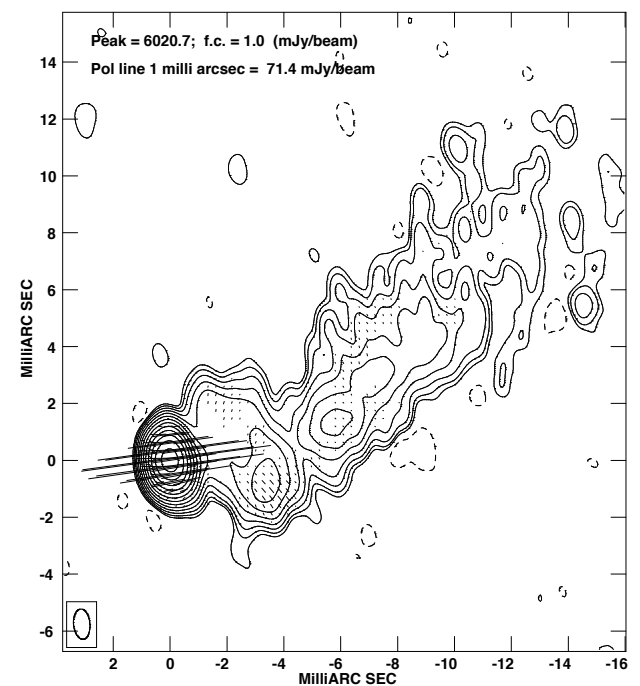

Figure 5. VLBA image at $15 \mathrm{GHz}$ of the parsec-scale structure of $3 \mathrm{C} 454.3$.

\section{The flat spectrum quasar $3 \mathrm{C} 454.3$}

This source, at redshift $z=0.859$, was the most active blazar in gamma rays during the first three years of Fermi observations. On the parsec scale its radio emission has a core-jet structure (Fig. 5). Since 2008, it has undergone three main gamma-ray outbursts $[1,3]$. In particular, in November 2010 it underwent an extraordinary 5day gamma-ray flare and became the brightest object in the gamma ray sky, 5 times brighter than the Vela pulsar [1]. After this major outburst the source entered in a quiet activity state at high energy, providing us with a unique opportunity for investigating a correlated variability between radio and gamma rays.

\subsection{The flux density variability}

To investigate the radio properties of the central region of 3C 454.3, we analysed MOJAVE data obtained between June 2009 and July 2013.

The analysis of the radio emission shows that the flux density was in a local minimum $\left(\mathrm{S}_{15 \mathrm{GHz}} \sim 6.4 \mathrm{Jy}\right)$ before the first gamma-ray flare detected in December 2009, and then it started to increase and reached its maximum $\left(\mathrm{S}_{15 \mathrm{GHz}} \sim\right.$ 27.6 Jy on mas-scale) in August 2010, about four months after a second strong gamma-ray flare that took place in April 2010 (Fig. 6). Unfortunately the time sampling of the observations is not adequate to investigate the flux density behaviour after the huge gamma-ray flare in November 2010. However, VLBI observations at higher frequencies pointed out an additional rising of the radio flux density after the November 2010 flare with the $43 \mathrm{GHz}$ increase leading on the $22 \mathrm{GHz}$, as expected in presence of opacity effects [e.g. 17, 22].

The analysis of multi-epoch 43-GHz VLBA observations could reveal the ejection of two superluminal jet components: one close in time with the December 2009 flare, and the other likely associated with the November 2010 [12]. No new superluminal component was observed associated with the April 2010 flare.

Since 2011 the source has been in a quiet activity state in gamma rays without any further flare episode. The same behaviour is observed in the $15-\mathrm{GHz}$ light curve, where the flux density has decreased to about $4 \mathrm{Jy}$. This result suggests that the variability at the two edges of the electromagnetic spectrum is tightly related, at least in this source. A different behaviour is observed, for example, in the radio-loud Narrow Line Seyfert 1 SBS 0846+513 where outbursts in the radio band are observed during quiet activity state in gamma rays (see Section 4) [7].

Thanks to the high resolution of the VLBA data, we could separate the core emission from that arising from the compact jet component that was ejected at the end of 2009 , close in time with the gamma-ray flare detected in December 2009. The analysis of the light curves of the core and the jet component shows that the flux density increase may be assigned to the knot component, which becomes even brighter than the unresolved core region (Fig. 7). This result is in agreement with the 43-GHz VLBA data presented in [12].

\subsection{The polarized emission}

An interesting behaviour is shown by the polarized emission. The polarized flux density reached its maximum 


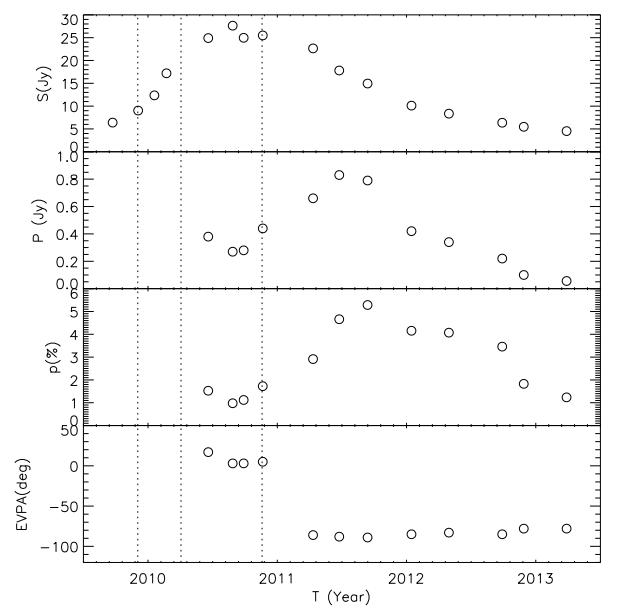

Figure 6. From top to bottom: total intensity flux density, polarization flux density, fractional polarization, and polarization angle for 3C 454.3, as derived from 15-GHz MOJAVE data. Vertical lines indicate the time of the gamma-ray flares.

when the total emission is already decreasing. Furthermore, after the November 2010 flare, the radio EVPA had an abrupt flip of almost $90^{\circ}$, switching from $\sim 5^{\circ}$ to $\sim 95^{\circ}$. This rotation may be due to the transition between the optically thick and thin regime. This interpretation is supported by observations at $43 \mathrm{GHz}$, where the $90^{\circ}$ flip of the EVPA was already observed in November 2009, when at $15 \mathrm{GHz}$ the EVPA was still $\sim 5^{\circ}$ [22] (Faraday rotation effects are negligible at these frequencies). From the analysis of the EVPA we find that the magnetic field is perpendicular to the jet axis. This is in agreement with the presence of a shock that is propagating along the jet and is ordering the component of the magnetic field which is perpendicular to the shock propagation. This may also explain the increase of the polarized emission, since the degree of polarization is related to the ordered component of the magnetic field which is enhanced by the passage of the shock.

\section{The NLSy1 SBS $0846+513$}

The radio source SBS $0846+513$ is associated with a Narrow Line Seyfert 1 galaxy at redshift $z=0.5835$. This is one of the five NLSy1s detected by Fermi-LAT. Its radio emission has a core-jet structure (Fig. 8). The source underwent three strong gamma-ray flares: one in June 2011, when the source was detected for the first time at high energies [7], and the other two in May and August 2012 [8].

The detection of gamma rays from this class of sources was unexpected, opening new questions on the physics in these objects [9].

To investigate the nature of this object we compare the gamma-ray light curve with the data at $15 \mathrm{GHz}$ from the Owens Valley Radio Observatory (OVRO). From this comparison we could not find any clear correlation between the variability in the two energy bands. Gamma-ray

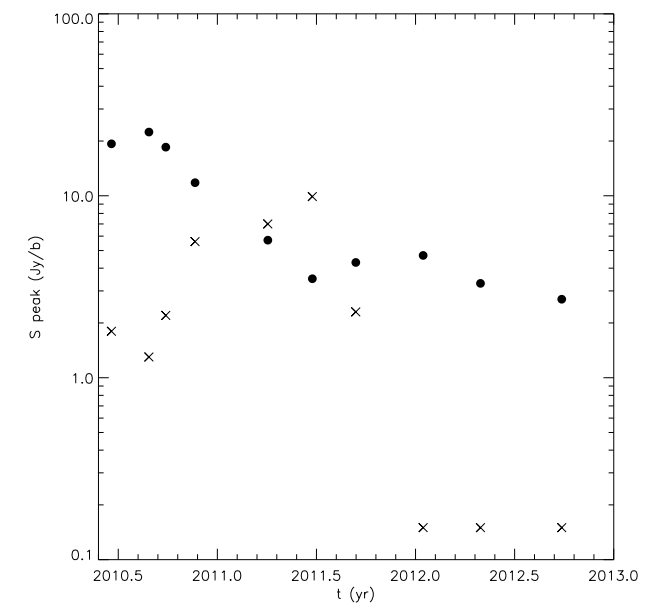

Figure 7. Peak flux density of the core component (filled circles), and of the jet knot (crosses) for 3C 454.3.

flares are not reliably associated with radio outbursts: only a slight increase of the flux density at $15 \mathrm{GHz}$ is observed a few months after the gamma ray flare. On the other hand, a large radio outburst occurred in December 2009, when the source was not detected in gamma rays. Interestingly, the analysis of the multi-epoch VLBA data from the MOJAVE programme pointed out that a superluminal jet component was ejected close in time with the radio outburst, representing an example of knot emission not connected to any gamma ray flaring activity [8].

A complicated behaviour is observed in 2012. Three radio outbursts occurring in May, October, and December 2012 were detected at $15 \mathrm{GHz}$ by OVRO, while only two gamma-ray flares, one in May and the other in August 2012, were observed. The most likely scenario suggests that the radio outbursts detected in October and December 2012 are likely the delayed counterparts of the two gamma-ray flaring episodes, while the radio flare observed in May has no clear counterpart at high energy [8].

\section{Concluding remarks}

Multiwavelength monitoring campaigns suggest a relation between gamma-ray flares and the radio variability, explained in terms of a shock moving along the jet, whose manifestation is a superluminal knot observable with highfrequency VLBI observations. However, the variability observed in gamma rays does not always show the same properties in the other energy bands, even if we consider the same source.

So far only a handful of the most powerful objects have been studied, and no statistically complete conclusions can be drawn. To better understand the physics at work in these objects, larger samples of objects spanning a wider luminosity range is needed.

With the jump in sensitivity provided by ALMA (more than an order of magnitude with respect to current interferometers working at same wavelengths) we will be able to study a large number of faint objects, both in total intensity 


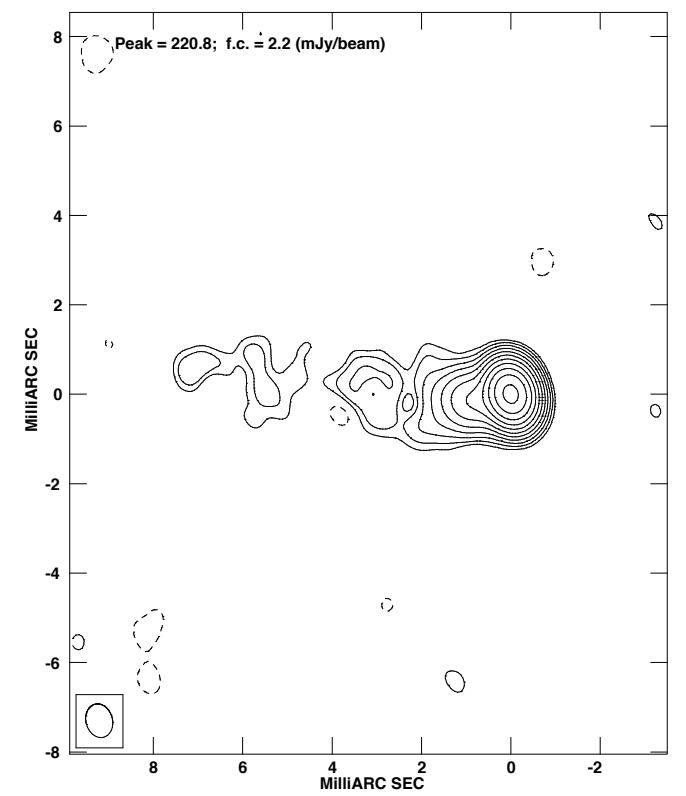

Figure 8. VLBA image at $15 \mathrm{GHz}$ of the NLSy1 SBS $0846+513$.

and polarization, which is fundamental for understanding the physics of high energy emitting radio sources.

\section{Acknowledgements}

The Fermi-LAT Collaboration acknowledges generous ongoing support from a number of agencies and institutes that have supported both the development and the operation of the LAT as well as scientific data analysis. These include the National Aeronautics and Space Administration and the Department of Energy in the United States, the Commissariat à l'Energie Atomique and the Centre National de la Recherche Scientifique / Institut National de Physique Nucléaire et de Physique des Particules in France, the Agenzia Spaziale Italiana and the Istituto Nazionale di Fisica Nucleare in Italy, the Ministry of Education, Culture, Sports, Science and Technology (MEXT), High Energy Accelerator Research Organization (KEK) and Japan Aerospace Exploration Agency (JAXA) in Japan, and the K. A. Wallenberg Foundation, the Swedish Research Council and the Swedish National Space Board in Sweden. Additional support for science analysis during the operations phase is gratefully acknowledged from the Istituto Nazionale di Astrofisica in Italy and the Centre National d'Études Spatiales in France. This research has made use of the data from the MOJAVE data base that is maintained by the MOJAVE team (Lister et al. 2009b). Data from the Steward Observatory spectropolarimetric project were used. This programme is supported by Fermi Guest Investigator grants
NNX08AW56G and NNX09AU10G.

\section{References}

[1] Abdo, A.A., Ackermann, M., Ajello, M., et al.; ApJ 733, 26 (2011)

[2] Abramowski, A., Acero, F., Aharonian, F., et al.; A\&A, 554, 107 (2013)

[3] Ackermann, M., Ajello, M., Baldini, L., et al.; ApJ 721, 1383 (2010)

[4] Ackermann, M., Ajello, M., Allafort, A., et al.; ApJ 743, 171 (2011)

[5] Cortina, J.; The Astronomer's Telegram, 3965 (2012)

[6] D’Ammando, F., Pucella, G., Raiteri, C.M., et al.; A\&A 508, 181 (2009)

[7] D’Ammando, F., Orienti, M., Finke, J., et al.; MNRAS 426, 317 (2012)

[8] D’Ammando, F., Orienti, M., Finke, J., et al.; MNRAS, in press, (2013)

[9] D’Ammando, F., Orienti, M., Finke, J., Larsson, J., Giroletti, M.; these proceedings (2013)

[10] Ghisellini, G., Madau P.; MNRAS 280, 67 (2003)

[11] Jorstad, S.G., Marscher, A.P., Marrox, J.R., et al.; ApJS 134, 181 (2001)

[12] Jorstad, S.G., Marscher, A.P., Smith, P.S., et al.; ApJ 773, 147 (2013)

[13] Lähteenmäki, A., Valtaoja, E.; ApJ 590, 95 (2003)

[14] Lister, M., Aller, H.D., Aller, M.F., et al.; AJ 137, 3718 (2009)

[15] Marscher, A.P., Jorstad, S.G., D’Arcangelo, F.D., et al.; Nature 452, 966 (2008)

[16] Marscher, A.P., Jorstad, S.G., Larionov, V.M., et al.; ApJ 710, 126

[17] Nagai, H., Kino, M., Niinuma, K., et al.; PASJ 65, 24 (2013)

[18] Orienti, M., Venturi, T., Dallacasa, D., et al.; MNRAS 417, 359 (2011)

[19] Orienti, M., Koyama, S., D’Ammando, F., et al.; MNRAS 428, 2418 (2013)

[20] Sikora, M., Modersky, R., Madejsky, G.M.; ApJ 675, 71 (2008)

[21] Smith P.S., Montiel E., Rightley S., et al.; eConf Proceedings C091122 (2009)

[22] Wehrle, A.E., Marscher, A.P., Jorstad, S.G., et al.; ApJ 758, 72 (2012) 